\title{
HOSTS OF INSECT EGG-PARASITES IN NORTH AND SOUTH AMERICA.
}

\section{By A. ARSÈne GIRAUlt, WAShington, D. C.}

THE following compilation of the hosts of the insect egg-parasites is made for several reasons, the most important of which is the labor which it saves by bringing together the much scattered rearing records of these insects. Other reasons are the importance of egg-parasites as a class, the aid given in identifying the parasites of any one host, and the interest which a compilation of this kind is likely to stimulate in the minds of economic entomologists for whom this list is primarily intended

The list considers the parasites from the standpoint of the host only, and it is not meant to bring out any important biologic facts concerning them, which should be considered rather in a list of the parasites and their hosts or host relations. Mainly, it answers but one question, "What parasites attack the egg of Carpocapsa pomonella, or other insect?" It is complete up to the end of the year 1906, and includes none but true, internal parasites of the egg, insects that pass their entire life-cycle within the egg of another insect excepting as adults. Insects that prey on the eggs, such as Pimpla, Anthomyia, Sarcophaga, Eunotus, often called egg-parasites, are not considered as such, and are therefore ignored; and parasites that oviposit on or into the egg, becoming fatal during some phase of postembryonic development of the host, through polyembryonic development, are also excluded, being considered parasites of the larva or pupa as the case may be. I consider Platygaster herrickii Packard a case in point.

The hosts are arranged alphabetically according to order, genus, and species, merely for the sake of convenience. A query preceding the parasite listed under any host, questions the record in one way or another; insects of doubtful generic position are enclosed within quotation marks New records are preceded by an asterisk. References to the literature are given in the most convenient way possible, and the nomenclature and synonymy are according to the latest authorities. Incomplete records are entirely omitted in the list, excepting in the case of a few important or suggestive ones. The record of the rearing of Ablerus clisiocampae Ashmead from the eggs of Malacosoma americana Fabricius has been proven to be undoubtedly incorrect, but in order to point out this mistake in as forcible a manner as possible, I have thought it wise to include it in the list of parasites of that host, there calling attention to the error. In this way, I doubly insure attention being drawn to the mistake. Anopedius error Fitch, erroneously recorded from the eggs of Nabis 
ferus Linnaeus, is treated in a like manner; it is a larval parasite of certain Diptera. And attention is also directed to the record of Elasmus albicoxa Howard from eggs of Basilarchia archippus Cramer, more or less discredited by both Howard and Ashmead (the latter, in litt.); and to the erroneous record of Hadronotus leptocorisa Howard on Leptocorisa tipuloides DeGeer (Ashmead, 1887 b, p. 119; id., 1893 a, p. 231). Polyneura (Webster, 1903, p. 33) should be Polynema, so Mr. Webster informs me. The list requires no further explanation.

Our knowledge of egg-parasites does not compare favorably with that of the parasites of the larva. But for the writings of Howard, Ashmead, and Riley and Howard, the recorded rearings would be very meagre indeed. It is mainly through the efforts of the two former, that so much is now known of this most important class of insect parasites, and we are at the present day much more advanced in this respect than are our European neighbors.

Egg-parasites are not difficult to rear, and being, perhaps, the most potent enemies of many of our injurious insects, it is desirable that greater care and more thoroughness be taken in rearing work; so that on account of their minute size, they are not overlooked.

In connection with this list, I mention some of the insects which are known to be hosts of egg-parasites, the latter still unknown. In the Lepidoptera, Anisota rubicunda Fabr., Euproctis chrysorrhoea Linn. (Europe), Junonia coenia Hübner, Malacosoma disstria Hübner, Megathymus yucce Boisd. et Leconte, Paonius excaecatus Smith et Abbot, Thorybes pylades Scudder, and Vanessa cardui Linn. have been recorded as being attacked by egg-parasites. Among the Hemiptera is Serinetha trivittata Say; the hymenopterus genus Hylatoma is probably attacked by the eulophid Hyperteles hylatomae Ashm. (Ashmead, 1888, Canadian Ent., XX, p. 105); while certain Odonata (Howard, 1901, Insect Book, p. 366) are also recorded to be hosts of egg-parasites. A species of "Trichogramma" probably attacks Hemerobius (Howard, Hymenoptera, in Stand. Nat. Hist., Boston, p. 511).

The insect egg-parasites of the Americas are all hymenopterous, as far as we know definitely at the present time. The majority of them belong to the superfamilies Chalcidoidea (mostly the families Trichogrammidae and Mymaridae, and the following: - the family Torymidae $<$ Podagrion only $>$; Eurytomidae $<$ Rileyini, - Macrorileya and Neorileya $>$; and the Encyrtidae $<$ Eupelminae,-Eupelmus, Anastatus; Encyrtinae,-Oöencyrtus, Dinocarsis $>$ and Proctotrypoidea (mostly the Scelioninae, comprising Phanurus, Te'enomus, Trissolcus, Aradophagus, Prosacantha, Caloteleia, Baryconus, Macroteleia, Cacus, Hadronotus, Idris, and Scelio in the tribes Telenomini, Teleasini, and Scelionini respectively, and the sub- 
family Ceraphroninae $<$ Aphanogmus only $>$. Outside of these two superfamilies, certain of the Evaniidae (namely Evania) of the superfamily Ichneumonoidea are also egg-parasites of the true insects, the cockroaches.

The most prominent and common of the genera of egg-parasites are undoubtedly Trichogramma and Telenomus, and of the species, Trichogramma pretiosa Riley is foremost, attacking no less than eleven or more hosts. Telenomus graptae Howard follows as the second most common species, attacking six hosts.

Other genera and doubtless families in the Chalcidoidea and Proctotrypoidea will be found to be parasitic on the eggs of American insects when we become more advanced in our knowledge of them. In Hawaii, for example, such has been found to be the case (Perkins, 1905, p. 200 et al; 1906), and also in Europe in a few cases. The foregoing enumeration of parasitic families and genera, therefore, cannot be said to be restrictive.

The known hosts of egg-parasites in continental North and South America comprise the following orders as listed: Coleoptera, Diptera, Hemiptera, Hymenoptera, Lepidoptera, Neuroptera, and Orthoptera, and in addition the Odonata. The families attacked in each order, where numerous, are widely separated, and in this respect the parasites seem to have very little choice. In order of being most attacked, in number of species, the Lepidoptera are first, then the Orthoptera, Hemiptera, Coleoptera and Hymenoptera.

\section{Hosts and their parasites.}

\section{Coleoptera.}

Host Parasite.

Attelabus bipustulatus Fabricius

Chlaenius impunctifrons Say

Conotrachelus nenuphar Herbst

Fidia viticida Walsh

Odontota dorsalis Thunberg

Pityopthorus consimilis Leconte Riley rault Howard
Poropoea species

Prosacantha caraborum

Anaphes conotracheli Gi-

Brachista fideae Ashmead

Fidiobia flavipes Ashmead

Trichogramma odontotae

? Aradophagus fasciatus Ashmead

querciperda Schwarz ? Aradophagus fasciatus Ashmead

Scarites subterraneous Fabricius

Prosacantha Riley

Tachypterus (Anthonomus) quad- ? Gonatocerus anthonomi rigibbus Say
Girault
Authority.

Hopkins, 1905, p. 132.

Ashmead, 1893a, pp. 180, 191, 451.

Girault, 1905, Ent. News, XVI, p. 220.

Webster, 1896, p. 69.

Slingerland, 1906, p. 93.

Chittenden, 1902, p. 82.

Ashmead, 1893a, p. 166.

Dalla Torre, 1898, p. 512. Cf. Ashmead 1893a, p. 166.

Riley \& Howard, 1891, p. 124.

Girault, 1905, Ent. News, XVI, p. 289. 
Diptera.

Tabanus atratus Fabricius

Anasa tristis DeGeer

A piomerus spissipes Say

Arilus cristatus Linnaeus

Ceresa bubalus Fabricius

Coccus hesperidum (Linnaeus)

Dysdercus suturellus HerrickSch.

Euschistus fissilis Uhler.

servus Say

tristigmus Say

Euthoctha galeator Fabricius Ashmead Ashmead

Howard

Ashmead

Ashmead novem. Howard

Brachyrhynchus granulatus Say Ashmead Ashmead Girault Ashmead Ashmead Howard Ashmead Ashmead Ashmead
Phanurus tabanivorus Hemiptera.

Eupelmus reduvii Howard Ashmead, 1887a, p. 190.

Hadronotus carinatifrons Girault, 1904, Ent. News, p.

Hadronotus rugosus

Hadronotus anasae

Oöencyrtus anasae

Hadronotus species

* Anastatus giraulti Ashmead MS.

Eupelmus reduvii Howard Howard, 1880, Can. Ent., XII,

* Oöencyrtus johnsoni

Aradophagus fasciatus

Trissolcus brochymenae

Telenomus perplexus

Trichogramma ceresara

Polynema (Cosmocoma) species novem.

? Trichogramma flava

? Hadronotus rugosus

* Trissolcus euschisti

Trissolcus euschisti

* Trissoleus euschisti

? Hadronotus rugosus Howard p. 208.

Riley \& Howard, 1891, p. 124.

Howard, 1888, p. 129.

Chittenden, 1899, p. 26.

Morgan, 1907, p. 54.

Girault, MS. notes, 1903.

Girault, MS. notes, 1905.

Dalla Torre, 1898, p. 512. Cf. Ashmead, 1893a, p. 166.

Girault, 1906, Psyche, XIII, p. 66.

Ashmead, 1888, Can. Ent. XX, p. 107.

Riley, 1894, p. 215.

Howard, 1888, p. 131.

Riley \& Howard, 1891, p. 124. Discredited by Ashmead, 1893a, pp. 232, 451.

Girault, MS. notes, 1905.

Ashmead, 1893a, pp. 162, 451.

Girault, MS. notes, 1905.

Ashmead, 1893a, pp. 232, 451.
Dalla Torre, 1898, p. 512. 
Homalodisca triquetra Fabricius

Icerya purchasi Maskell

Largus succinctus Linnaeus

Lepidosaphes ulmi (Linnaeus)

Liburnia species

Metapodius femoratus Fabricius

Murgantia histrionica Hahn.

Nabis ferus Linnaeus

Pentatoma ligata Say sayi Stål

Podisus modestus Dallas

Podisus maculiventris Say

Thyantha custator Fabricius

Tibicen septendecim Linnaeus

Zelus bilobus Say

longipes Linnaeus
Ooctonus homalodiscae Ashmead

? Phanurus opacus Howard

? Alaptus iceryae Riley

Hadronotus largi Ashmead

? Anaphes gracilis Howard

Anagrus columbi Perkins

Hadronotus floridanus Ashmead

Oöencyrtus johnsoni Howard

Trissolcus murgantiae Ashmead podisi Ashmead

? Anopedius error Fitch

Telenomus ashmeadi Morrill.

Telenomus podisi Ashmead

Hadronotus species nov.

? Telenomus dimmocki Ashmead

Telenomus podisi Ashmead

Trissolcus podisi Ashmead

? Trissolcus thyantae Ashmead

Trissolcus thyantae Ashmead

A phanogmus floridanus Ashmead

Lathromeris cicadae Howard

Hadronotus leptocorisae Howard

Hadronotus leptocorisae Howard
Sanderson, 1906, p. 51.

Ashmead, 1893a, p. 142.

Riley, 1888, Ins. Life, I, p. 130.

Dalla Torre, 1898, p. 498.

Howard, 1888, p. 130.

Perkins, 1905, p. 198.

Riley \& Howard, 1891, p. 124.

Johnson, 1900, p. 21.

Morgan, 1897, pp. 140, 141, 159.

Riley, 1880, pp. 196-197. Cf. Ashmead, 1893a, p. 291.

Morrill, 1907, p. 10.

Ashmead, 1893a, p. 451. Cf. ib., p. 159.

E. Potts, 1891, Ent. News, II, pp. 53-54.

Dimmock, 1897, p. 148.

Ashmead, 1893a, p. 159.

Riley \& Howard, 1891, p. 124.

Dalla Torre, 1898 , p. 512. Cf. Ashmead, 1893a, p. 163.

Ashmead, 1893a, p. 163.

Riley \& Howard, 1891, p. 123. Queried by Ashmead, 1893a, p. 451.

Howard, 1898, Can. Ent., XXX, pp. 102-103.

Riley \& Howard, 1891, p. 124. Cf. Ashmead, 1893a, p. 231, \& 1887 b. p. 119.

Ashmead, 1887b, p. 119. 


\section{Hymenoptera.}

Anthophorabia species

Caliroa (Selandria) obsoleta Norton

Eriocampoides limacina Retzius (= Selandria cerasi Peck)

Isosoma captivum Howard

grande Riley

hordei Harris

tritici Fitch

Pachynematus palliventris

Cresson

Pteronus ribesii Scopoli

Aglais milberti Godart

Alabama argillacea Hübner

Alsophila pometaria Harris

Anosia plexippus Linnaeus

Automeris io Fabricius

Autographa brassicae Riley

Basilarchia archippus Cramer

Bellura gortynides Walker (= densa Walker)
Packardiella putnamii

Packard

Trichogramma minuta $\quad$ Morgan, 1897, pp. 144, 159.

Riley

"Encyrtus" species Marlatt, 1897, p. 6.

? Oligosita americana $\quad$ Webster, 1903, p. 35.

Ashmead

? Oligosita americana Webster, 1903, p. 22.

Ashmead

Oligositaamericana

Ashmead

Polynema ((?) Polyneura)

citripes Ashmead

? Olvgosita americana Webster, 1903, p. 22.

Ashmead

? Trichogramma pretiosa Riley

Trichogramma pretiosa Riley

\section{Lepidoptera.}

Trichogramma intermedia Howard

Trichogramma pretiosa Riley

"Platygaster" species

* Telenomus gnophaelae Ashmead

Trichogramma intermedia Howard

Eupelmus species

Trichogramma pretiosa Riley

Trichogramma minuta Riley

Trichogramma minutissima Packard

? Elasmus albicoxa

Howard

Telenomus arzamae Riley
Scudder, 1889, I, p. 429.

Riley, 1879, Can. Ent., XI, pp. 161-162.

Riley. Packard \& Thomas, 1883, p. 176.

Girault, MS. notes, 1905.

Howard, 1889, p. 1895.

Howard, 1891, p. 568.

Howard, 1888, p. 124.

Fletcher, 1893, p. 160 .

Scudder, 1889, I, p. 279.

Howard, 1885, p. $30 . \quad C f . \quad i d$. , Ins. Life, IV, p. 253.

Ashmead, 1893a, pp. 158, 450. 
Calpodes ethilius Cramer

Carpocapsa pomonella (Linnaeus)

Chlorippe clyton Boisduval \& Leconte

Datana interregima Grote \& Robinson ministra Drury

Diacrisia virginica Fabricius

Euvanessa antiopa Linnaeus

Gnophaela latipennis Boisduval

Heliothis obsoleta Fabricius

Hemerocampa leucostigma Smith \& Abbot

Heodes hypophleas Boisduval

Heterocampa bilineata Packard

Hyphantria cunea Drury textor Harris

Ianassa lignicolor Walker

Laphygma frugiperda Smith \& Abbot,

Malacosoma americana Fabricius ? Ablerus clisiocampae A chalcid

Trichogramma pretiosa Riley

Telenomus gossypiicola Ashmead

Eupelmus species

Telenomus spilosomatis Ashmead

Telenomus graptae Howard

Telenomus gnophaelae Ashmead

Telenomus heliothidis Ashmead

Trichogramma pretiosa Riley

Trichogramma pretiosa nigra Girault

Trïhogramma fraterna Fitch ${ }^{1}$

Trichogramma orgyiae Fitch ${ }^{1}$

Telenomus graptae Howard

Telenomus graptae Howard

Trichogramma species

Trichogramma pretiosa Riley

Trichogramma pretiosa Riley
"Trichogramma" species.

Telenomus rileyi Howard Riley \& Howard, 1891, p. 123.

Telenomus orgyiae Fitch

Telenomus bifidus Riley

Telenomus bifidus Riley Ashmead ${ }^{2}$
Morgan, 1897, pp. 149, 159.

Howard, 1891, p. 568. vide Dimmock, 1885, p. 279. Riley \& Howard, 1891, p. 123.

Bruner, 1890, p. 103.

Riley \& Howard, 1891, p. 123.

Riley \& Howard, 1891, p. 123 . cf. Mally, 1893, p. 25.

Howard, 1888, p. 124.

Girault, 1906.

Riley, 1887a, p. $32 . \quad C f$. Howard, 1897, pp. 7, 30-31, 52, \& Hulst. 1889, p. 205. vide Packard, 1890, p. 265.

Dalla Torre, 1898, p. 3.

Scudder, 1889, II, p. 1008.

Howard, 1894, p. 280.

Riley, 1887 b, p. 531.

Bruner, 1890, p. 43.

Riley \& Howard, 1891, p. 124.

Dyar, 1893, p. 256.

Riley, 1885a, p. 104.

Ashmead, 1893b, p. 10.

${ }^{1}$ For the validity and synonymy of these two species, $c f$. Riley, 1887, p. 32, Packard, 1890, p. 265 , and Howard, 1897, p. 7 .

2 Undoubtedly incorrect; a parasite of various species of Coccidæ, cf. Howard, 1894, p. 6, and others. 
Malacosoma (Clisiocampa) species

Mamestra picta Harris

Melalopha inclusa Hübner

Mompha (Laverna) luciferella Clemens

Notolophus antiqua Linnaeus

Notolophus (Orgyia) species

Oeneis macounii Edwards

Paleacrita vernata Peck

Paonıus myops Smith \& Abbot

Papilio glaucus Linnaeus

Papilio glaucus turnus Linneaus

Phlegethontias sexta Johanssen

Platynota rostrana Walker

Polychrosis viteana Clemens ${ }^{1}$

Polygonia interragationis Fabricius

progne Cramer
"Pteromalus" species

"Platygaster" species

Telenomus clisiocampae Riley

Telenomus clisiocampae Riley

Telenomus heliothidis Ashmead

Telenomus species novem

Trichogramma pretiosa Riley

Telenomus ichthyurae Ashmead

Telenomus lavernae Ashmead

Telenomus orgyiae Fitch

Telenomus californicus Ashmead

Telenomus orgyiae Fitch

Trichogramma intermedia Howard

"Platygaster" species

? Eupelmus species

Trichogramma minutissima Packard

Trichogramma interme- $\quad$ Fletcher, 1893, p. 160. dia Howard

Trichogramma minutissi- $\quad$ Fletcher, 1893, p. 160. ma Packard

Telenomus sphingis Ashmead

Trichogramma pretiosa Riley

Trichogramma pretiosa Riley

* Trichogramma pretiosa Riley

Telenomus graptae Howard

Trichogramma intermedia Howard

Telenomus graptae Howard
Packard, 1880, p. 207, fig. 139. Glover, 1877, p. 102.

Ashmead, 1893a, pp. 160, 450.

Ashmead, 1893a, p. 160.

Davis, 1893, p. 26.

Fletcher, 1893, p. 161.

Fletcher, 1893, p. 161.

Riley \& Howard, 1891, p. 123.

Riley \& Howard, 1891, p. 123.

Howard, 1897, p. 30.

Ashmead, 1893a, pp. 150, 450.

Ibid., p. 153.

Howard, 1889, p. 1895.

Riley, 1870b, p. 103.

Howard, 1891, p. 568.

Scudder, 1889, II, p. 1304.

Ashmead, 1893a, pp. 156, 450.

Ashmead, 1887c, p. 17.

Hubbard, 1885, p. 153.

Johnson, Fred, MS. notes, 1906.

Riley \& Howard, 1891, p. 123.

Howard, 1889, III, p. 1895.

Howard, 1889, III, p. 1891.

1 North East, Penn. 
Sanninoidea exitiosa (Say)

Schizura leptinoides Grote

Sphida (Arzama) obliqua Walker

Telea polyphemus Cramer

Thanaos lucilius Lintner

Therına fervidaria Hübner, somniaria Hulst

Thymelicus cernes Boisduval \& Leconte

Thyridopteryx ephemeraeformis Haworth

Vanessa atalanta Linnaeus

Chrysopa perla (Linnaeus) species

Amblycorypha oblongifolia

DeGeer

Blatta orientalis Linnaeus

Blattella germanica (Linnaeus)

Chortophaga viridifasciata DeGeer

Dichromorpha viridis Scudder Dictyopterus reticulatus Thunberg

Dissosteira carolina Linnaeus

Ischnoptera pennsylvanica DeGeer
Telenomus species

Telenomus chrysopae Ashmead

\section{Orthoptera.}

Telenomus quaintancei

Girault

Telenomus caelodasidis Ashmead mead

Eupelmus species

Trichogramma intermedia Howard

Telenomus species novem

Telenomus graptae

Howard

Dinocarsis thyridopterygis Ashmead

Trichogramma minutissima Packard

\section{Neuroptera.}

Anastatus mirabilis

Walsh.

Evania appendigaster (Linnaeus)

Evania species novem

Evania appendigaster (Linnaeus)

? Scelio oedipodae Ashmead

? Scelio hyalinipennis

Ashmead

Scelio species

? Scelio fuscipennis Ashmead

Scelio ovivorus Riley

Evania dorsalis (Westwood)
Girault, 1906, Psyche, XIII, p. 64.

Ashmead, 1893a, p. 159.

Riley \& Howard, 1891, p. 123.

Dimmock, 1897, p. 148.

Howard, 1891, p. 568.

Scudder, 1889, II, p. 1467.

Fletcher, 1893, p. 160.

Scudder, 1889, II, p. 1731.

Ashmead, 1886, p. 98.

Howard, 1889, p. 1895.

Howard, 1888, p. 123.

Ashmead, 1893a, pp. 159, 451.

Riley, 1870a, pp. 296-297, 369370.

Marlatt, 1902, p. 11.

Cockerell, 1894, p. 209.

Marlatt, 1902, p. 11.

Morgan, 1901, p. 24.

Morgan, 1901, p. 24.

Warner, 1903, pp. 308-309.

Ashmead, 1893a, p. 243.

cf. Dalla Torre, 1898, p. 495.

Riley \& Howard, 1891, p. 124.

Ashmead, 1900, p. 7. 
Mantis brasiliana Linnaeus

\author{
Melanoplus atlantis Riley \\ differentialis \\ Thomas \\ species \\ spretus Thomas
}

Microcentrum retinerve Burmeister

Oecanthus fasciatus Fitch latipennis Riley

nigricornis Walker, niveus DeGeer

species

"Oedipoda" species

Orchelimum agile DeGeer

glaberrimum Burmeister

Periplaneta americana Linnaeus

australasiae Fabricius

Schistocerca americana Drury

melanocera Stål obscura Fabricius
Podagrion melleum West- Dalla Torre, 1898, p. 369. wood

Scelio calopteni Riley Ashmead, 1893a, pp. 246, 451.

Scelio hyalinipennis Ash- Morgan, 1901, p. 23. mead

Scelio oedipodae Ashmead Morgan, 1901, p. 23.

Scelio luggeri Riley Riley \& Howard, 1891, p. 124.

? Scelio ovivorus Riley pp. 305-306. Cf. Psyche, IV, 1883, p. 78.

Anastatus mirabilis Walsh Hubbard, 1885, pp. 134-135.

Anastatus mirabilis Walsh Ashmead, 1894, p. 245.

Cacus oecanthi Riley

Caloteleia species

"Rileya" species Riley \& Howard, 1891, p. 124. Bruner, 1890, p. 126, footnote. Bruner, 1890, p. 126, footnote.

Anastatus mrabilis Walsh, Felt, 1906, p 699.

Baryconus oecanthi Riley Dalla Torre, 1898, p. 501.

Cacus oecanthi Riley

Caloteleia species

Idris species

"Rileya" species

Macrorileya oecanthi

Ashmead

Scelio oedipodae Ashmead Dalla Torre, 1898, p. 495.

Eupelmus xiphidii Ashmead

Macroteleia sp., near floridana Ashmead

Macroteleia floridana Ashmead

Macroteleia virginiensis Ashmead

"Eulophus" species Evania appendigaster (Linneaus)

"Pteromalus" species Evania appendigaster (Linnaeus)

? Scelio hyalinipennis Ashmead

? Scelio oedipodae Ashmead

Scelvo ernstri Riley

Scelio hyalinipennis Ashmead

Dalla Torre, 1898, p. 500.

Bruner, 1890, p. 126 , footnote.

Webster, 1891, pp. 345-346.

Bruner, 1890, p. 126, footnote.

Ashmead, 1904, p. 264.

Morgan, 1901, p. 31.

Morgan, 1901, p. 31.

Ashmead, 1893a, p. 451. Cf. pp. 217-218.

Ashmead, 1893a, p. 218.

Westwood, 1839, p. 423.

Ashmead, 1900, p. 7.

Westwood, 1839, p. 423.

Ashmead, 1900, p. 7.

Morgan, 1901, p. 24.

Morgan, 1901, p. 24.

Riley \& Howard, 1891, p. 124.

Morgan, 1901, p. 28. 


$\begin{array}{cll}\text { peregrina } \text { Olivier } & \begin{array}{l}\text { Scelio oedipodae Ashmead } \\ \text { Scelio ernstii Riley }\end{array} & \begin{array}{l}\text { Morgan, 1901, p. } 28 . \\ \text { Riley, 1885b, p. } 22 .\end{array} \\ \text { Stagmomantis carolina Linnaeus } & \begin{array}{c}\text { Podagrion mantis } \\ \text { Ashmead }\end{array} & \text { Morgan, 1897, p. } 159 . \\ \text { limbata } \text { Saussure } & \begin{array}{c}\text { ? Podagrion mantis } \\ \text { Ashmead }\end{array} & \text { H. S. Barber, 1906, In litt. }\end{array}$

\section{Acknowledgments.}

The compilation and arrangement of this list were first suggested by Dr. F. H. Chittenden, Bureau of Entomology, U. S. Department of Agriculture. I desire to thank Messrs. Otto Heidemann and A. N. Caudell, United States National Museum, for their kindness in correcting the nomenclature of the Hemiptera and Orthoptera respectively, and Dr. Wm. H. Ashmead of the Museum, and Mr. F. M. Webster of the Bureau of Entomology, for valuable information on various points. I would also like to acknowledge the courtesy of Mr. E. A. Schwarz of the National Museum, for untangling questions of nomenclature and synonymy in the Coleoptera.

\section{LITERATURE REFERRED TO.}

1839. Westwood, John Obadiah. Introduction Modern Classification Insects, I 1870. a Riley, Charles Valentine. American Ent. and Botanist, St. Louis, II (1869-1870).

b 2nd Rep. State Ent. Missouri.

1877. Glover, Townsend. Rep. U. S. Ent. f. 1877.

1878. Riley, C. V., Alpheus Spring Packard, \& Cyrus Thomas. 1st Rep. U. S. Ent. Commission, Washington.

1880. Packard, Alpheus Spring. Guide Study Insects, New York, edit. 7.

Riley, Charles Valentine. Rep. Ent. f. 1879, U. S. Dep. Agric.

1883. Riley, C. V., A. S. Packard, and C. Thomas.-3rd Rep. U. S. Ent. Commission, Washington.

1885. Dimmock, Anna K. Psyche, IV.

Howard, Leland Ossian. Bull. No. 5, old series, U. S. Dep. Agric., Bureau Entomology.

Hubbard, Henry Guernsey. Insects Affecting Orange, U. S. Dep. Agric., Div. Ent.

a Riley, Charles Valentine. 4th Rep. U. S. Ent. Commission, Washington.

b. Riley, Chales Valentine. Proc. Ent. Soc. Washington, I. 
1886. Ashmead, William Harris. Canadian Ent. XVIII.

1887. a Ashmead, William Harris. Trans. American Ent. Soc., XIV.

b Idem. Ent. Americana, III.

c Idem. Bull. No. 14, old series, U. S. Dep. Agric., Div. Ent.

a Riley, Charles Valentine. Bull. No. 10, old series, U. S. Dep. Agric., Div. Ent.

b Idem. Rep. Ent., in Rep. U. S. Commissioner Agric. f. 1886.

1888 Howard, Leland Ossian. Proc. Ent. Soc. Washington, I.

1889. Idem. In Scudder, 1889, III.

Hulst, George D. 9th Annual Rep. New Jersey Agric. Exp. Sta. f. 1888.

Scudder, Samuel H. Butterflies Eastern U. S. and Canada, Cambridge, I-III.

1890. Bruner, Lawrence. Bull. No. 14, III, Nebraska Agric. Exp. Sta.

Packard, Alpheus Spring. 5th Rep. U. S. Ent. Commission, Washington.

1891. Howard, Leland Ossian. Proc. U. S. National Museum, Washington, XIV.

Riley, Chas. Valentine, and Leiand Ossian Howard. Insect Life

U. S. Dep. Agric., Div. Ent., IV.

Webster, Francis Marion. Ibidem. III.

1893. a Ashmead, William Harris. Bull. No. 45, U. S. National Museum.

b Idem. Proc. Ent. Soc. Washington, III.

Davis, G. C. Bull. No. 102, Michigan Agric. Exp. Sta.

Dyar, Harrison Gray. Canadian Ent., XXV.

Fletcher, James. Rep. Ent. and Botanist f. 1892, Experimental Farms Canada.

Mally, F. W. Bull. No. 29, old series, U. S. Dep. Agric., Div. Ent.

Riley, Charles Valentine, and Leland Ossian Howard. Insect Life, U. S. Dep. Agric., V.

1894. Ashmead, William Harris. Insect Life, U. S. Dep. Agric., VII.

Cockerell, Theodore Drew Alison. Ibidem, VII.

Howard, Leland Ossian. Ibidem, VII.

Riley, Charles Valentine. Rep. Ent. f. 1893, U. S. Dep. Agric.

1895. Ashmead, William Harris. Bull. Illinois State Laby. Natural History, Urbana, IV.

1896. Slingerland, Mark Vernon. Bull. No. 6, new series, U. S. Dep. Agric., Div. Ent.

Webster, Francis Marion. Ibidem. 
1897. Dimmock, George. Proc. Ent. Soc. Washington, IV.

Howard Leland Ossian. Bull. No. 5, tech. series, U. S. Dep. Agric. Div. Ent.

Marlatt, Charles Lester. Circ. No. 26, 2 series, U. S. Dep. Agric. Div. Ent.

Morgan, H. A. Bull. No. 48, 2d series. Louisiana Agric. Exp. Sta.

1898. Datlla Torre, Carl G. de. Catalogs Hymenopterorum, V.

Osborn, Herbert. Bull. No. 16, new series, U. S. Dep. Agric., Div. Ent.

1899. Chittenden, Frank Hurlbut. Bull. No. 19, new series, U. S. Dep. Agric., Div. Ent.

1900. Ashmead, William Harris. Proc. U. S. National Museum, XXIII

Johnson, Willis G. Bull. No. 26, new series, U. S. Dep. Agric., Div. Ent.

1901. Morgan, H. A. Bull. No. 30, new series, U. S. Dep. Agric., Div. Ent.

1902. Chittenden, Frank Hurlbut. Bull. No. 38, new series, U. S. Dep. Agric., Div. Ent.

Marlatt, Charles Lester. Circ. No. 51, 2d series, U. S. Dep. Agric., Div. Ent.

1903. Warner,_- Proc. Ent. Soc. Washington, V.

Webster, Francis Marion. Bull. No. 42, U. S. Dep. Agric., Div. Ent.

1904. Ashmead, William Harris. Memoir Carnegie Museum, Pittsburgh, I, No. 4 (Serial No. 25).

1905. Hopkins, Andrew Delmar. Proc. Ent. Soc. Washington, VII.

Perkins, R. C. L. Bull. No. 1, Div. Ent., Hawaiian Sugar Planter's Association, Honolulu.

1906. Girault, Alecandrè Arsìne. Canadian Ent. XXXVIII, p. 81.

Felt, Ephraim Porter. Memoir No. 8, N. Y. State Museum, Albany, II.

Perkins, R. C. L. Bull. No. 1, Div. Ent., Hawaiian Sugar Planter's Association, Honolulu, pp. 241.

Sanderson, E. Dwight. Bull. No. 57, U. S. Dep. Agric., Bureau Ent.

Silingerland, Mark Vernon. Bull. No. 235, Cornell Agric. Exp. Sta., Ithaca.

1907. Morgan, A. C. Bull. No. 63, Pt. IV, U. S. Dep. Agric., Bureau Ent.

Morrill, A. W. Bull. No. 64, Pt. I, U. S. Dep. Agric. Bureau Ent. 

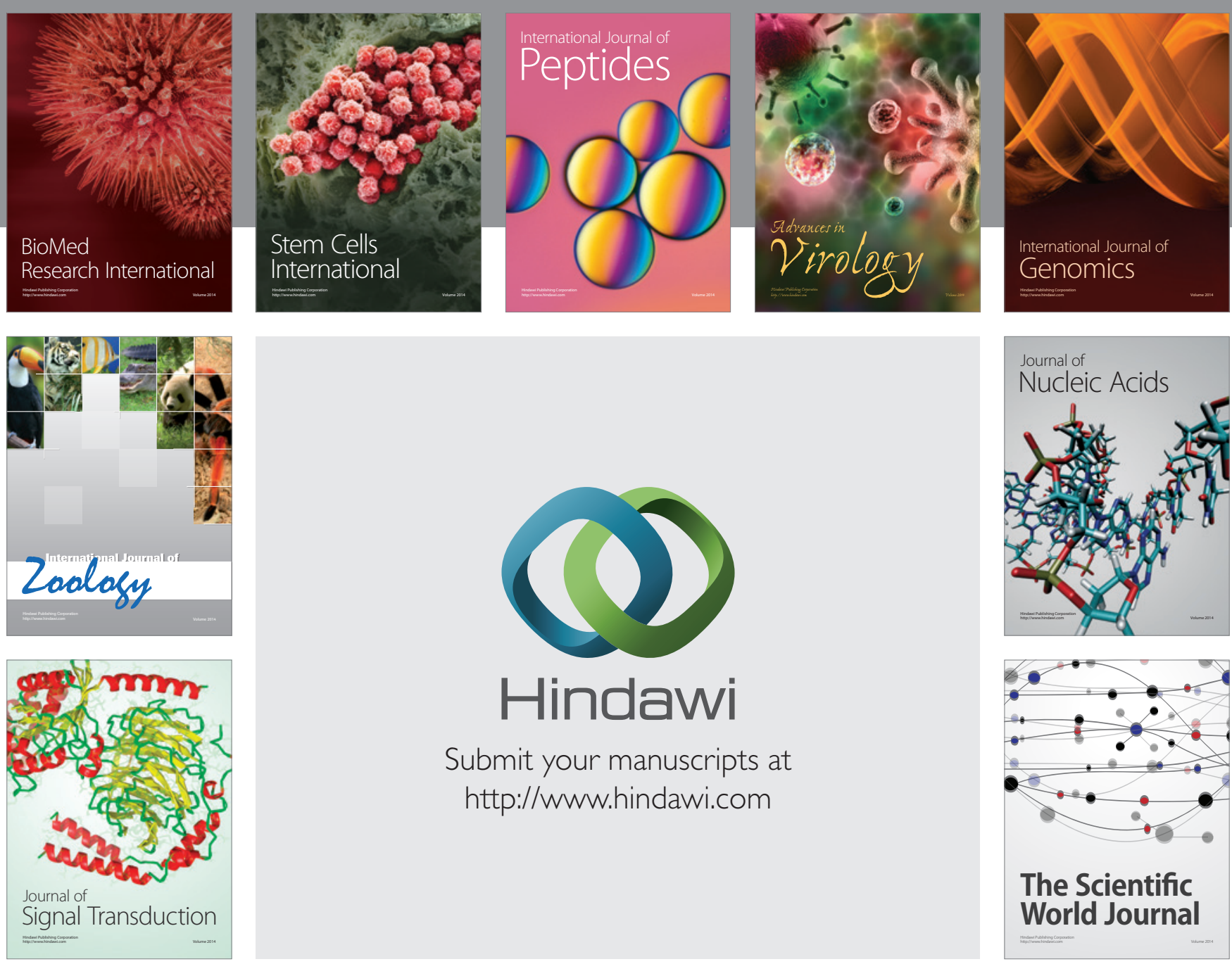

Submit your manuscripts at

http://www.hindawi.com
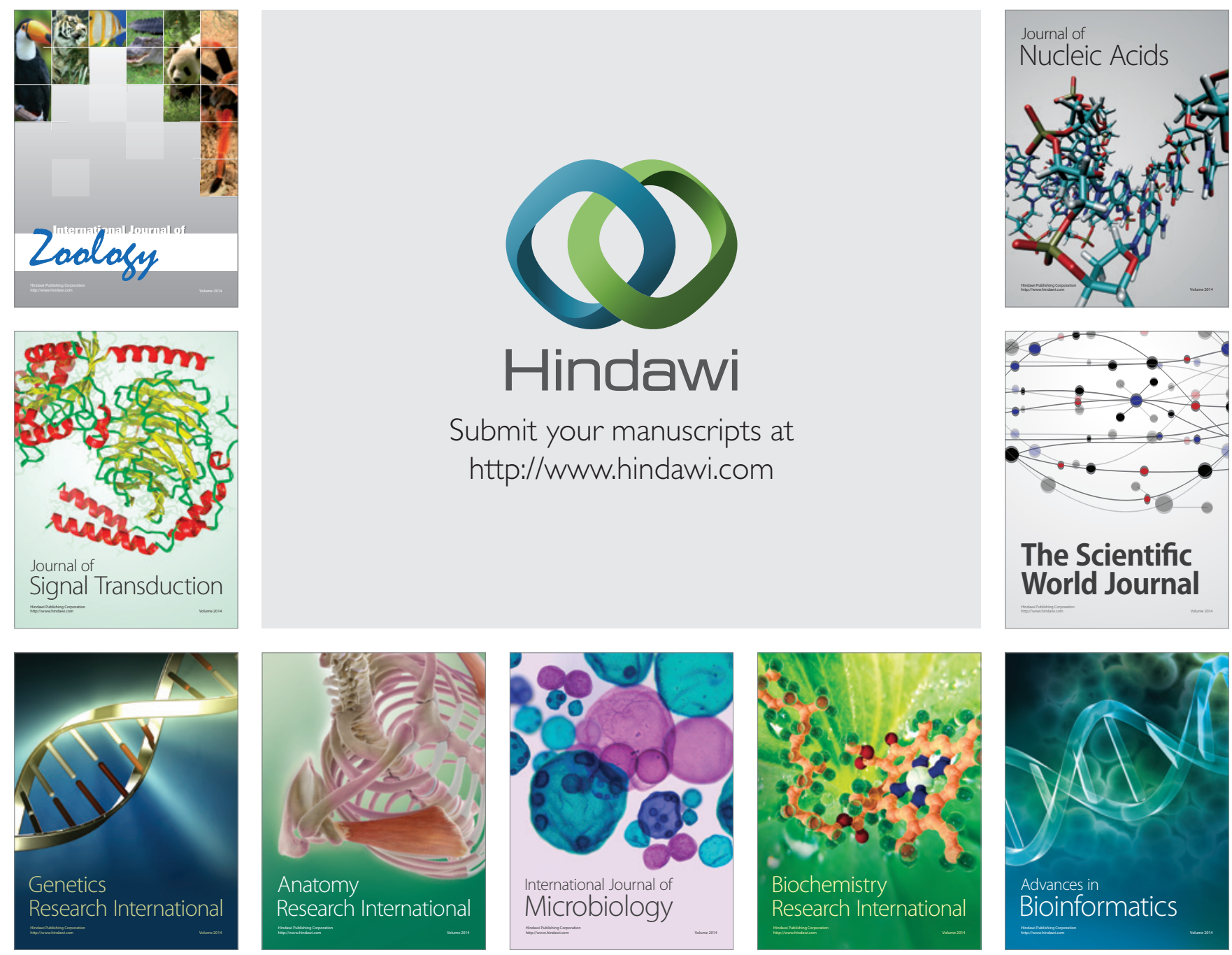

The Scientific World Journal
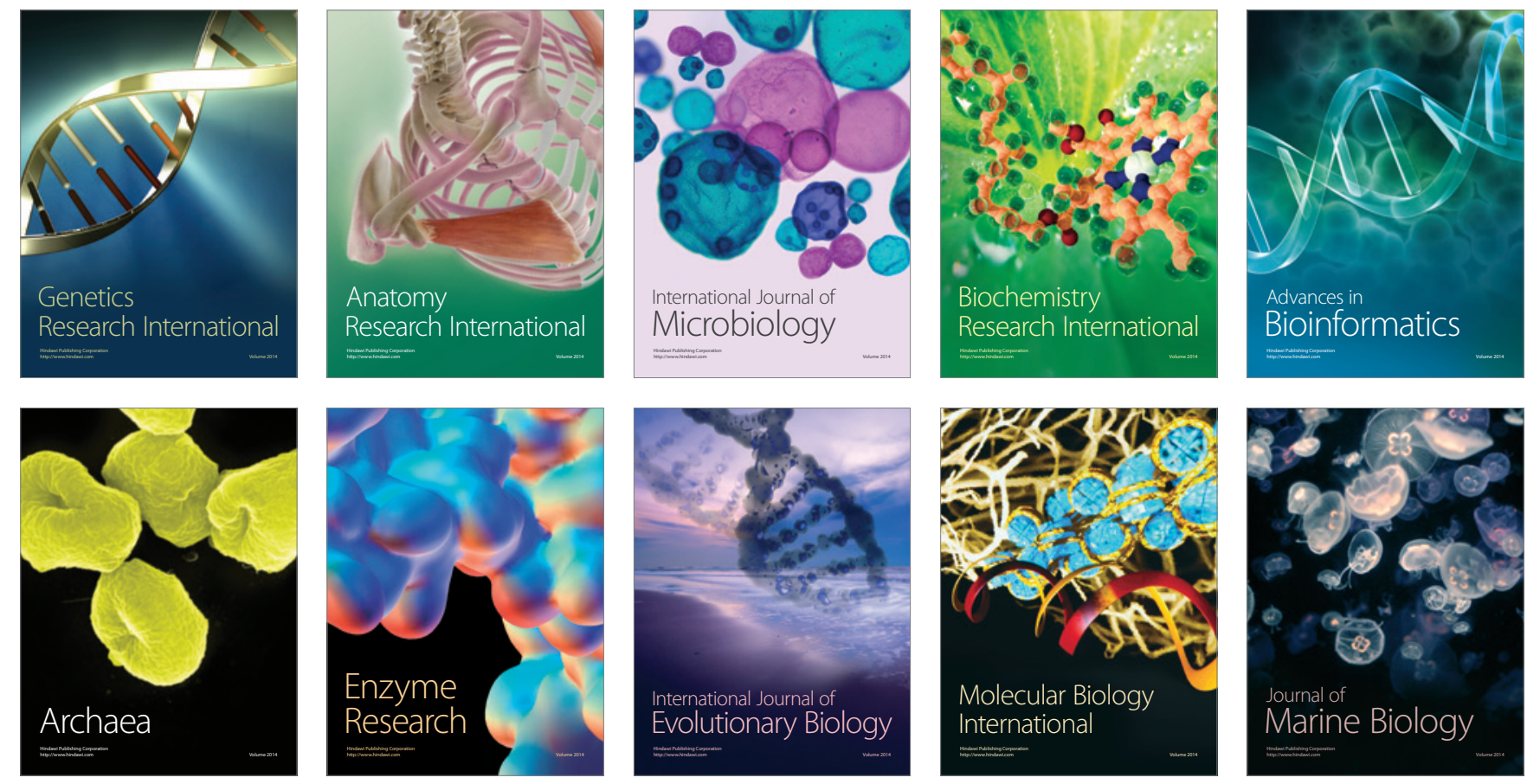